\title{
A HIGH INDICES THEOREM WITHOUT A NONTRIVIAL SOLUTION
}

\author{
BO I. JOHANSSON
}

\begin{abstract}
In this paper we will extend the high indices Theorem by Hardy-Littlewood, which says that an Abel summable series, where the exponents fulfil a Hadamard gap criterion, is convergent and it converges to its Abel sum. The focus here is concerning a class of summability methods and their critical rate of convergence, i.e. for a given summability method what is its rate of convergence implying that the series must be identically constant.
\end{abstract}

\section{Introduction}

In this article we consider different summability methods of a series. As an example we have the well known Abel summability which says: For a series $\sum_{n=1}^{\infty} a_{n}$ to be Abel summable with the sum 0 we mean that $\lim _{x \rightarrow 0^{+}} \sum_{n=1}^{\infty} a_{n} e^{-p_{n} x}=0$, where $p_{n}=n$ usually. Under what conditions this implies that the original series converges to zero was first studied by Tauber. Since then there has been an extensive study of generalizations of similar problem settings. Here we rather study for what rate of decay certain summability methods force the coefficients in the series to be identically zero. Also, we restrict the indices $\left\{p_{n}\right\}_{n=1}^{\infty}$ to be a Hadamard sequence, i.e., the indices satisfy the gap condition $p_{n+1} / p_{n} \geq \delta>1$ for all consecutive indices. The case $p_{n}=n$ has been studied in [7] and [6]. In the article Remarks to a paper by D. Gaier on gap theorems, see [3], Hálasz proved the following result. In fact, he had a less restricted gap condition.

Theorem A. If

$$
\sum_{n=1}^{\infty} a_{n} e^{-p_{n} x}=O\left(e^{-s(x)}\right) \text { as } x \rightarrow 0^{+}, \quad p_{n+1} / p_{n} \geq \delta>1,
$$

where $s$ is a convex function satisfying

$$
\int_{0}^{1} \sqrt{-s^{\prime}(x)} d x=\infty
$$

Received 18 April 2008. 
then

$$
a_{n}=0 \quad \text { for } \quad n=1,2, \ldots
$$

In this article our goal is to generalize the above result by including a class of summability methods. Our result in the case of Abel summability is the next result. We have

THEOREM 1. If

$$
\sum_{n=1}^{\infty} a_{n} e^{-p_{n} x}=O\left(e^{-s(x)}\right) \text { as } x \rightarrow 0^{+}, \quad p_{n+1} / p_{n} \geq \delta>1,
$$

where $s$ is a twice differentiable function satisfying

$$
\int_{0}^{1} \sqrt{-s^{\prime}(x)} d x=\infty
$$

and

$$
x\left(x s^{\prime}(x)\right)^{\prime} \geq a>0
$$

for $x>0$, then

$$
a_{n}=0 \quad \text { for } \quad n=1,2,3, \ldots
$$

Above we have referred to Abel summability, i.e., we have considered the exponential kernel, $x \mapsto e^{-x}, x>0$. There are other interesting kernels such as the Lambert kernel $x \mapsto x e^{-x} /\left(1-e^{-x}\right), x>0$, and the Rényi kernel $x \mapsto e^{-x} /\left(1+e^{-x}\right), x>0$. The main result in this article can also be applied to those summability methods. To exemplify this, we state the following result which essentially is a direct application of Theorem 4 . We have

THEOREM 2. If

(5) $\sum_{n=1}^{\infty} a_{n} \frac{e^{-p_{n} x}}{1+e^{-p_{n} x}}=O\left(e^{-s(x)}\right)$ as $x \rightarrow 0^{+}, \quad p_{n+1} / p_{n} \geq \delta>e^{\pi / u_{o}}$,

where $u_{o}=\min \left\{u>0 ; \zeta\left(\frac{1}{2}+i u\right)=0\right\}$ and $s$ is a twice differentiable function satisfying

$$
\int_{0}^{1} s(x) d x=\infty
$$


and

$$
x\left(x s^{\prime}(x)\right)^{\prime} \geq a>0
$$

for $x>0$, then

$$
a_{n}=0 \quad \text { for } \quad n=1,2,3, \ldots
$$

REMARK. We know that $u_{o}=14.1347 \ldots$ From this we get $e^{\pi / u_{o}}=$ $1.2489 \ldots$.

We could equally well have formulated Theorem 2 in terms of the Lambert kernel instead of the Rényi kernel, as we have done, and still got the same result. More generally, an interesting class of functions falling into the frame of this paper will be the family $\mathscr{K}$, where

$$
\begin{aligned}
\mathscr{K}=\{f \in C(0, \infty) & ; f(x)=x^{\rho} \sum_{m=1}^{\infty} \alpha_{m} \exp (-m x), \\
& \left.\rho \geq 0, \alpha_{m}=O\left(m^{\gamma}\right), \gamma<\infty, f \neq 0, f^{\prime} \in L^{1}(0, \infty)\right\} .
\end{aligned}
$$

\section{Preliminaries}

Before we formulate our result we like to reformulate the problem by transforming it to the real axis in the following way, i.e., formula (1) becomes

$$
\sum_{n=1}^{\infty} a_{n} \int_{\log p_{n}-x}^{\infty} \frac{d}{d y}\left(-e^{-e^{y}}\right) d y=O\left(e^{-s\left(e^{-x}\right)}\right) \quad \text { as } \quad x \rightarrow \infty
$$

where the the sequence $\left\{p_{n}\right\}$ satisfies $\log p_{n+1}-\log p_{n} \geq \log \delta>0$. Here we have transformed the positive real axis to the real axis by the inverse function of $x \mapsto e^{-x}, x>0$. A general setting with $f \in \mathscr{K}$ and $k(x)=\frac{d}{d x}\left(-f\left(e^{x}\right)\right)$, $x \in \mathrm{R}$, will be

$$
\sum_{n=1}^{\infty} a_{n} \int_{\lambda_{n}-x}^{\infty} k(y) d y=O\left(e^{-s\left(e^{-x}\right)}\right) \quad \text { as } \quad x \rightarrow \infty
$$

where the the sequence $\left\{\lambda_{n}\right\}$ satisfies $\lambda_{n+1}-\lambda_{n} \geq \delta>0$. The kernel connected to Abel summability as in formula (1) is $k(x)=\exp (-\exp (x)+x)$, where $x \in \mathrm{R}$. The kernel $k(x)=e^{-e^{x}+x} /\left(1+e^{-e^{x}}\right)^{2}, x \in \mathrm{R}$ is connected to the summability method in Theorem 2 .

We now introduce another class of kernels. After the natural transformation the three kernels in the introduction, among many others, are included in this class. Next we use the notation $k_{\alpha}(x)=k(x) \exp (\alpha x), x \in \mathrm{R}$. 
Definition 3. Suppose that $k$ is a function such that $k_{\alpha} \in L^{1}(\mathrm{R})$ for some $\alpha \in \mathrm{R}$, and that its Fourier transform $\hat{k}_{\alpha}(u)=\int_{-\infty}^{\infty} k_{\alpha}(x) \exp (-i u x) d x$, $u \in \mathrm{R}$, has an extension, which is analytic in the open upper halfplane and continuous in the closed one, and that $\hat{k}_{\alpha}^{\prime}(u)$ exists for $u \in \mathrm{R}$. Let $\beta>0$ and $\xi$ be real, and suppose further that

$$
\begin{aligned}
& \max _{|\xi| \leq \beta}\left|\frac{\hat{k}_{\alpha}(u+\xi)}{\hat{k}_{\alpha}(u)}\right| \leq \exp (\theta(u)), \\
& \max _{|\xi| \leq \beta}\left|\frac{\hat{k}_{\alpha}(w+\xi)}{\hat{k}_{\alpha}(w)}\right| \leq C \exp (C|w|), \quad \Im(w) \geq 0
\end{aligned}
$$

and

$$
\left|\frac{\hat{k}_{\alpha}^{\prime}(u)}{\hat{k}_{\alpha}(u)}\right| \leq \exp (\theta(u)),
$$

where $C$ is a constant, $\theta(u)$ is a positive even function of $u$, monotonically increasing for $u>0$ and

$$
\int_{1}^{\infty} \frac{\theta(u)}{u^{2}} d u<\infty
$$

Then we say that $k \in \mathscr{L}_{\alpha}$.

This definition was introduced in [4].

\section{The main result}

In this section we state our main result, which forces a restriction for the rate of convergence for a summability method with a non-identically zero series. The two theorems stated in the introduction are corollaries of this result. In fact, we have a general result concerning identically vanishing high indices series. It can be formulated as follows:

THEOREM 4. Let $\left\{\lambda_{n}\right\}$ be a sequence of positive numbers satisfying

$$
\lambda_{n+1}-\lambda_{n} \geq c>0, \quad n=1,2,3, \ldots
$$

Suppose there exists an $\alpha \geq 0$ and an $\epsilon_{o}>0$ such that $k \in \mathscr{L}_{\alpha}$ and that

$$
\exp (-(\alpha+\epsilon) x) \sum_{n=1}^{\infty} a_{n} \int_{\lambda_{n}-x}^{\infty} k(y) d y
$$

converges uniformly on every set $\left\{x: x<x_{o}\right\}, x_{o}<\infty$ and for $\epsilon \in\left[0, \epsilon_{o}\right]$, and defines a bounded function on R. Suppose further that

$$
\hat{k}_{\alpha} \text { has a meromorphic extension into the lower halfplane, }
$$



on every line $\Im(w)=\sigma, \sigma \leq \alpha$, there exists a closed interval I such that $\hat{k}(u+i \sigma) \neq 0$ if $u \in I$, where $|I|=2 \pi / c$,

$$
(1 / \hat{k})\left(-i\left(\beta_{o}+m \beta\right)\right)=0, \quad \beta>0, \quad \beta_{o}>-\beta, m=1,2,3, \ldots,
$$

and

$$
(1 / \hat{k})(u+i v)=O\left(\exp (|v| \eta(|v|)), \quad v \rightarrow-\infty, \quad|u| \leq \frac{2 \pi}{c},\right.
$$

where $\eta(v)$ is nondecreasing. Let $q(x)$ be a positive and twice differentiable function with

$$
q^{\prime \prime}(x) \geq \delta>0 \quad \text { for } \quad x \geq 0
$$

and satisfying

$$
\int^{\infty} \exp \left(-\frac{\beta}{2}\left(x+\eta\left(q^{\prime}(x)\right)\right)\right) q^{\prime \prime}(x) d x=\infty .
$$

Then

$$
\sum_{n=1}^{\infty} a_{n} \int_{\lambda_{n}-x}^{\infty} k(y) d y=O(\exp (-q(x))), \quad x \rightarrow \infty
$$

implies that

$$
a_{n}=0, \quad n=1,2,3, \ldots
$$

In order to prove the above theorem, we need some known results. We formulate them in the next six lemmas. Let us start by defining a specific function and use a theorem by Fuchs, see [2], to get our result. We have

Lemma 5. The function

$$
H(z)=\prod_{k=1}^{\infty} \frac{\beta_{o}+k \beta-z}{\beta_{o}+k \beta+z} \exp \left(\frac{2 z}{\beta_{o}+k \beta}\right), \quad \beta>0, \quad \beta_{o}>-\beta,
$$

is analytic in $\Re(z) \geq 0$ and satisfies there

$$
|H(z)| \geq(B|z|)^{2 \Re(z) / \beta}
$$

for all $z$ outside circles of radius $\beta / 3$ with centers at the points $\left\{\beta_{o}+k \beta\right\}_{k=1}^{\infty}$, where $B$ is a constant. 
The next lemma is formulated and proved by Ostrowski in [9]. It can be written as follows.

LEMMA 6. Let $f \in C^{\infty}[0, \infty)$ be a non-identically vanishing function satisfying

$$
f^{(n)}(0)=0, \quad n=0,1,2, \ldots,
$$

and

$$
\sup _{x \geq 0}\left|f^{(n)}(x)\right| \leq m_{n} \quad \text { for } \quad n=0,1,2, \ldots
$$

A necessary and sufficient condition for the existence of such a function $f$ is that

$$
\int_{1}^{\infty} \log T(r) \frac{d r}{r^{2}}<\infty,
$$

where $T(r)=\sup _{n \geq 1} \frac{r^{n}}{m_{n}}$.

In order to use Lemma 6 we need an equivalent way to verify the condition (20). For that purpose we introduce the concept of Faberian minorant for a function $m$, which is the largest function $m^{*}$ fulfilling both the conditions $m(\lambda) \geq m^{*}(\lambda)$ for all $\lambda \geq 0$ and $\log m^{*}(\lambda) / \lambda$ is monotonically increasing for $\lambda \geq 0$. Particularly, $\frac{\log m^{*}(\lambda)}{\lambda}=\inf _{\mu \geq \lambda} \frac{\log m(\mu)}{\mu}$. In our case we restrict the sequence $\left\{m_{n}\right\}$ in the above Lemma to be increasing. Also, let $m$ be an increasing function satisfying $m(n)=m_{n}$ for all non-negative integers $n$.

Further, we introduce the function $\beta(\lambda)=\sqrt[\lambda]{m^{*}(\lambda)}$ for $\lambda \geq 0$ and its (pseudo)inverse $\rho(r)=\inf \{\lambda ; \beta(\lambda) \geq r\}$. We can now formulate more easily handled equivalent conditions than the one in (20). This result is also included in the same paper [9] by Ostrowski. We have

LEMMA 7. The convergence of

$$
\int^{\infty} \log T(r) \frac{d r}{r^{2}}
$$

is equivalent to the convergence of both the integrals

$$
\int^{\infty} \frac{\rho(r)}{r^{2}} d r \quad \text { and } \quad \int^{\infty} \frac{d \rho}{\beta(\rho)} .
$$

Our interest in Lemma 6 is its negative part, i.e., that part where the integral in (20) is divergent. From Lemma 7 this is true if anyone of the two last integrals diverges, especially the last one. This is used in the next lemma, which is a fundamental part in the proof of our main theorem. We get 
Lemma 8. Suppose $f(z)$ is an analytic function in $\Re(z)>0$ satisfying

$$
f\left(\beta_{o}+k \beta\right)=0, \quad k=1,2,3, \ldots, \quad \beta>0, \quad \beta_{o}>-\beta
$$

and

$$
|f(x+i y)| \leq C \exp (\theta(x)), \quad x \geq 0,
$$

for some increasing function $\theta$. Suppose further that

$$
\int^{\infty} \exp \left(-\frac{\beta}{2} \frac{\theta^{*}(x)}{x}\right) d x=\infty
$$

where $\theta^{*}$ is the greatest minorant of the function $\theta$ satisfying $x \mapsto \theta^{*}(x) / x$, $x>0$, is monotonically increasing.

Then

$$
f=0 \text {. }
$$

Proof. Let us define a function $\tilde{\Phi}$ by

$$
\tilde{\Phi}(z)=f(z)(H(z))^{-1}(1+z)^{-2}, \quad \Re(z)>0,
$$

where $H$ is the function constructed in Lemma 5. From the definition of the function $H$, see formula (18), and property (21) for the function $f$, it follows that the function $\tilde{\Phi}(z)$ is analytic for $\mathfrak{R}(z)>0$. Using the estimates in (19) and (22), we conclude that $\tilde{\Phi}(x+i y)$ is bounded by

$$
|\tilde{\Phi}(x+i y)| \leq C e^{\theta(x)}(B r)^{-\frac{2 x}{\beta}}\left(1+r^{2}\right)^{-1} \quad(r=|x+i y|) .
$$

Because of the inequality (26), we can form a function $\Phi(s)$ defined for $s \geq 0$ by

$$
\Phi(s)=\frac{1}{2 \pi i} \int_{x-i \infty}^{x+i \infty} \tilde{\Phi}(z) s^{2 z / \beta} d z \quad(x>0) .
$$

Since the above integral obviously is independent of $x$, let $x \geq n \cdot \beta / 2$. Then we can calculate the $\mathrm{n}$ :th derivative of $\Phi(s)$. From (27) it follows that

$$
\Phi^{(n)}(s)=\frac{1}{2 \pi i} \int_{x-i \infty}^{x+i \infty} \tilde{\Phi}(z) \frac{2 z}{\beta}\left(\frac{2 z}{\beta}-1\right) \ldots\left(\frac{2 z}{\beta}-n+1\right) s^{\frac{2 z}{\beta}-n} d z
$$


Using the estimate (26) in the above formula, we get

$$
\begin{aligned}
\left|\Phi^{(n)}(s)\right| & \leq \frac{C}{2 \pi} e^{\theta(x)} B^{-\frac{2 x}{\beta}} \int_{-\infty}^{\infty} r^{-\frac{2 x}{\beta}}\left(\frac{2 r}{\beta}\right)^{n} s^{\frac{2 x}{\beta}-n} \frac{d y}{1+r^{2}} \\
& =\frac{C}{2 \pi} e^{\theta(x)} B^{-\frac{2 x}{\beta}}\left(\frac{2}{\beta}\right)^{n} s^{\frac{2 x}{\beta}-n} \int_{-\infty}^{\infty} r^{n-\frac{2 x}{\beta}} \frac{d y}{1+r^{2}} .
\end{aligned}
$$

For $x>n \beta / 2$ the inequality (28) implies immediately that

$$
\Phi^{(n)}(0)=0, \quad n=0,1,2, \ldots
$$

Letting $x=n \beta / 2$ in the estimate (28), we get

$$
\max _{s \geq 0}\left|\Phi^{(n)}(s)\right|=O\left(\left(\frac{2}{\beta B}\right)^{n} e^{\theta\left(\frac{n \beta}{2}\right)}\right) .
$$

Using Lemma 6 combined with Lemma 7, it follows from (29) and (30) that

$$
\Phi(s)=0, \quad s \geq 0,
$$

because

$$
\int^{\infty} \exp \left(-\frac{1}{x} \theta^{*}\left(\frac{x \beta}{2}\right)\right) d x=\frac{2}{\beta} \int^{\infty} \exp \left(-\frac{\beta}{2 x} \theta^{*}(x)\right) d x=\infty,
$$

where $\theta^{*}$ is the largest minorant of the function $\theta$ satisfying that $x \mapsto \theta^{*}(x) / x$, $x>0$, is increasing. But since the function $\tilde{\Phi}(z), \Re(z)>0$, is the Mellin transform of $s \mapsto \Phi\left(s^{-\beta / 2}\right)$, i.e.,

$$
\tilde{\Phi}(z)=\int_{0}^{\infty} \Phi\left(s^{-\beta / 2}\right) s^{z-1} d s,
$$

it follows that the function $\tilde{\Phi}$ must be identically zero. From the definition of $\tilde{\Phi}$ in (25) it implies that $f$ is identically zero, i.e., (24) is true which completes the proof of the lemma.

The next lemma is the starting point in the proof of Theorem 4 and it is formulated as follows. For a proof see [4].

Lemma 9. Let $\left\{\lambda_{n}\right\}$ be a sequence of positive numbers satisfying

$$
\lambda_{n+1}-\lambda_{n} \geq c>0, \quad n=1,2,3, \ldots
$$

Suppose there exists an $\alpha \geq 0$ such that $k \in \mathscr{L}_{\alpha}$ and that

$$
\exp (-(\alpha+\epsilon) x) \sum_{n=1}^{\infty} a_{n} \int_{\lambda_{n}-x}^{\infty} k(y) d y
$$


converges uniformly on every set $\left\{x: x<x_{o}\right\}, x_{o}<\infty$ and defines a bounded function on $\mathrm{R}$. Then

$$
a_{n}=O\left(\exp \left(\alpha \lambda_{n}\right)\right), \quad n=1,2,3, \ldots
$$

We also need a theorem by Pólya, see Levinson [8] Theorem XXIX. This result is stated in the next lemma.

LEMMA 10. Let

$$
h(w)=\sum_{n=1}^{\infty} a_{n} e^{i w \lambda_{n}}
$$

be a Dirichlet series. Suppose $\left\{\lambda_{n}\right\}_{n=1}^{\infty}$ satisfies

$$
\lambda_{n+1}-\lambda_{n} \geq c>0 \text {. }
$$

Then on the axis of convergence the function $h$ has at least one singularity in every closed interval of length $2 \pi / c$.

Now we are ready to prove the main result in this paper, i.e., to prove Theorem 4.

Proof of Theorem 4. Let

$$
F(x)=\sum_{n=1}^{\infty} a_{n} \int_{\lambda_{n}-x}^{\infty} k(y) d y .
$$

First we observe that the conditions (8) and (9) combined with the fact that $k \in \mathscr{L}_{\alpha}$ imply, from Lemma 9 , that the coefficients $\left\{a_{n}\right\}_{n=1}^{\infty}$ satisfy

$$
a_{n}=O\left(\exp \left(\alpha \lambda_{n}\right)\right), \quad n=1,2,3, \ldots
$$

The above estimate can be found in an article by Johansson, see [4]. Moreover, it follows directly from (8) and (31) in combination with the fact that $k \in \mathscr{L}_{\alpha}$, that the function

$$
w \mapsto \hat{k}(w) h(w),
$$

is analytic for $\Im(w)>\alpha$, where $w \mapsto h(w)$ is the function defined by the Dirichlet series

$$
h(w)=\sum_{n=1}^{\infty} a_{n} e^{i w \lambda_{n}}, \quad \Im(w)>\alpha .
$$

The fact that (9), (14) and (16) hold implies that the Fourier transform $w \mapsto$ $\hat{F}(-w)$ is analytic for $\Im(w)<\alpha+\epsilon_{o}$. Furthermore, combining the above with 
condition (9) and $k \in \mathscr{L}_{\alpha}$, more precisely $k_{\alpha} \in L^{1}(\mathrm{R})$, we easily see that in the strip $\alpha<\Im(w)<\alpha+\epsilon_{o}$ the relation

$$
\hat{F}(-w)=\frac{i \hat{k}(w) h(w)}{w}
$$

holds. Using the fact that $w \mapsto \hat{k}(w) h(w)$ is analytic in the halfplane $\Im(w)>\alpha$ and $w \mapsto F(-w)$ is analytic in the halfplane $\Im(w)<\alpha+\epsilon_{o}$ implies that the equality in (33) can be extended to the entire complex plane. This, in combination with (10) implies that $h(w)$ has a meromorphic extension into $\Im(w) \leq \alpha$. But the sequence $\left\{\lambda_{n}\right\}$ satisfies (8), so from Lemma 10 it follows that the Dirichlet series (32) must have a pole in each closed interval of length $2 \pi / c$ on the axis of convergence. The condition (11) and formula (33) then says that $h(w)$ has an analytic extension as a Dirichlet series into the entire complex plane.

Next, we want to estimate the growth of the function $v \mapsto h(u+i v)$ as $v \rightarrow-\infty$, by using

$$
\frac{1}{C}\left(\sum\left|a_{n} e^{-v \lambda_{n}}\right|^{2}\right)^{\frac{1}{2}} \leq\left(\int_{I}|h(u+i v)|^{2} d u\right)^{\frac{1}{2}} \leq C\left(\sum\left|a_{n} e^{-v \lambda_{n}}\right|^{2}\right)^{\frac{1}{2}},
$$

where $I$ is an interval with length $|I|>2 \pi / c$, and $C$ is a constant only depending on $I$ and $\left\{\lambda_{n}\right\}$. For a proof of (34) see [5]. Let us fix the interval $I$ centered around the origin. First, we want to estimate the growth of the Dirichlet series $h(u+i v)$ in the $u$-direction. We start with

$$
h(u+i v)=\int_{0}^{u} h^{\prime}(s+i v) d s+h(i v) .
$$

Without loss of generality, suppose $u$ is positive, and let $n$ be the smallest integer greater than $u /|I|$. We get

$$
\begin{aligned}
|h(u+i v)| & \leq \sum_{k=0}^{n-1} \int_{k|I|}^{(k+1)|I|}\left|h^{\prime}(s+i v)\right| d s+|h(i v)| \\
& \leq \sum_{k=0}^{n-1}|I|^{1 / 2}\left(\int_{k|I|}^{(k+1)|I|}\left|h^{\prime}(s+i v)\right|^{2} d s\right)^{1 / 2}+|h(i v)| \\
& \leq n|I|^{1 / 2} \max _{0 \leq k \leq n-1}\left\|h^{\prime}(\cdot+i v)\right\|_{L^{2}(k|I|,(k+1)|I|)}+|h(i v)| \\
& \leq 2 u|I|^{-1 / 2} \max _{0 \leq k \leq n-1}\left\|h^{\prime}(\cdot+i v)\right\|_{L^{2}(k|I|,(k+1)|I|)}+|h(i v)|
\end{aligned}
$$


and

$$
\begin{aligned}
\left(\int_{k|I|}^{(k+1)|I|}\left|h^{\prime}(s+i v)\right|^{2} d s\right)^{1 / 2} & \leq C\left(\sum\left|a_{n} \lambda_{n} e^{-v \lambda_{n}}\right|^{2}\right)^{\frac{1}{2}} \\
& \leq C\left(\sum\left|a_{n} e^{\lambda_{n}} e^{-v \lambda_{n}}\right|^{2}\right)^{\frac{1}{2}} \\
& \leq C^{2}\left(\int_{I}|h(s+i(v-1))|^{2} d s\right)^{1 / 2} \\
& \leq C^{2}|I|^{1 / 2}\|h(\cdot+i(v-1))\|_{L^{\infty}(I)} .
\end{aligned}
$$

Thus

$$
|h(u+i v)| \leq 2 C^{2}|u| \max _{u \in I}|h(u+i(v-1))|+|h(i v)| .
$$

In order to complete the proof, we need an estimation of the growth of $\hat{F}(u+i v)$ as $v \rightarrow \infty$. From (14) it follows particularly that $q^{\prime}(x)$ is strictly increasing. Thus, $q^{\prime}$ is invertible, i.e., the function $\gamma=\left(q^{\prime}\right)^{-1}$ exists. Using (14) once more together with (16) we get

$$
\begin{aligned}
|\hat{F}(u+i v)| & \\
= & \left|\int_{-\infty}^{\infty} F(x) e^{-i(u+i v) x} d x\right| \\
& \leq \int_{-\infty}^{0}|F(x)| e^{v x} d x+\int_{0}^{\infty}|F(x)| e^{v x} d x \\
= & o(1)+O\left(\int_{0}^{\infty} \exp (-q(x)+v x) d x\right) \\
= & O\left(\int_{-\gamma(v)}^{\infty} \exp (-q(x+\gamma(v))+v(x+\gamma(v))) d x\right) \\
= & O\left(\int _ { - \gamma ( v ) } ^ { \infty } \operatorname { e x p } \left(-q(\gamma(v))-x q^{\prime}(\gamma(v))\right.\right. \\
& \left.\left.\quad-\frac{x^{2}}{2} q^{\prime \prime}(\gamma(v)+\kappa x)+v x+v \gamma(v)\right) d x\right), \quad 0<\kappa=\kappa(x)<1 .
\end{aligned}
$$

In the above estimate we used a Taylor expansion of the function $q(\gamma(v)+\cdot)$. 
The fact that $q^{\prime \prime}(\gamma(v)+\kappa x) \geq \delta>0$ can be used next in order to get

$$
\begin{aligned}
|\hat{F}(u+i v)| & =O\left(\exp (v \gamma(v)) \int_{-\infty}^{\infty} \exp \left(-\frac{\delta}{2} x^{2}\right) d x\right) \\
& =O(\exp (v \gamma(v))) \quad \text { as } \quad v \rightarrow \infty .
\end{aligned}
$$

Let $|u| \leq 2 \pi / c$. Using the condition (13) and the estimate (36) in the representation (33), we get

$$
\begin{aligned}
|h(u-i v)| & =\left|i(u-i v) \frac{\hat{F}(-u+i v)}{\hat{k}(u-i v)}\right| \\
& =O(v \exp (v \gamma(v)+v \eta(v))), \quad \text { as } \quad v \rightarrow \infty .
\end{aligned}
$$

The inequality in (35) combined with the above equality implies that we get the following uniform estimate for $u \in$ R, i.e., (37)

$$
\left|\frac{h(u-i v)}{(u-i(v+1))^{2}}\right|=O(\exp ((v+1)(\gamma(v+1)+\eta(v+1)))), \quad \text { as } \quad v \rightarrow \infty .
$$

Moreover, since the function $\hat{F}$ is entire and the Fourier transform $\hat{k}$ satisfies condition (12), the equality (33) implies that we get

$$
\frac{h(w)}{(w-i)^{2}}=0 \text { for } w=-i\left(\beta_{o}+m \beta\right), \quad \text { with } \quad m=1,2,3, \ldots
$$

Considering the function $h /(\cdot-i)^{2}$, we see that the above zero-set corresponds to (21) in Lemma 8. The same function also satisfies the estimate (37), which is similar to (22) with $\theta=i d \cdot(\gamma+\eta)$. Since $v \mapsto \frac{\theta(v)}{v}=\gamma(v)+\eta(v)$ is an increasing function as a consequence of the facts that $\gamma$ is the inverse function of a strictly increasing function combined with (13), we can choose $\theta^{*}=\theta$ in Lemma 8 because $\theta$ is definitively the greatest minorant of itself.

Hence, we have

$$
\begin{aligned}
\int^{\infty} \exp \left(-\frac{\beta}{2} \frac{\theta^{*}(v)}{v}\right) d v & =\int^{\infty} \exp \left(-\frac{\beta}{2}(\gamma+\eta)(v)\right) d v \\
& =\int^{\infty} \exp \left(-\frac{\beta}{2}\left(x+\eta\left(q^{\prime}(x)\right)\right)\right) q^{\prime \prime}(x) d x=\infty
\end{aligned}
$$

which follows from (15) and establish the final condition (23) in Lemma 8 in order to conclude the fact that the function $h$ is identically zero. From this it trivially follows that (17) is true and the proof is completed. 


\section{Application}

In this section we will see that Theorem 1 and Theorem 2 respectively are simple corollaries of Theorem 4 . To see this we start by considering the expression

$$
f(t)=\sum_{n=1}^{\infty} a_{n} f\left(p_{n} t\right)=O\left(e^{-s(t)}\right) \quad \text { as } \quad t \rightarrow 0^{+},
$$

for a general kernel $f \in \mathscr{K}$, where the function class $\mathscr{K}$ was introduced in the beginning of this paper. The sequence $\left\{p_{n}\right\}_{n=1}^{\infty}$ is satisfying $p_{n+1} / p_{n} \geq \delta$ for $n=1,2,3, \ldots$, and for some $\delta>1$. Such a sequence is called a Hadamard sequence. The function $s=q(-\log (\cdot))$, where $q$ satisfies condition (14). Of special interest here is the exponential kernel together with the kernels by Rényi and Lambert. Those are

$$
\begin{aligned}
& f_{1}(t)=e^{-t}, \\
& f_{2}(t)=t e^{-t} /\left(1-e^{-t}\right)
\end{aligned}
$$

and

$$
f_{3}(t)=e^{-t} /\left(1+e^{-t}\right), \quad t>0 .
$$

After a transformation of the series in (38) to our generic form as in (7), by $k(x)=\frac{d}{d x}\left(-f\left(e^{x}\right)\right)$ and $x \in \mathrm{R}$, it is easily seen that the kernels above have the Fourier transforms

$$
\begin{aligned}
& \hat{k}_{1}(u)=\Gamma(1-i u) \\
& \hat{k}_{2}(u)=-i u \zeta(1-i u) \Gamma(1-i u)
\end{aligned}
$$

and

$$
\hat{k}_{3}(u)=\left(1-2^{1+i u}\right) \zeta(-i u) \Gamma(1-i u)
$$

for $u \in \mathrm{R}$. Here $\Gamma$ is Euler's $\Gamma$-function and $\zeta$ is Riemann's $\zeta$-function.

First we consider the exponential kernel $f_{1}$. Its belonging Fourier transform $\hat{k}_{1}$ is given above. We know that $1 / \Gamma$ is analytic in the entire complex plane and is non-zero except for the non-positive integers, i.e.

$$
\left(1 / \hat{k}_{1}\right)(-i m)=0, \quad m=1,2,3, \ldots
$$

We also know that

$$
\left(1 / \hat{k}_{1}\right)(u-i v)=O(\exp (v \log v)),
$$

for $|u| \leq C$ and $v \rightarrow \infty$, where $C$ is any finite constant. Another fact about the behaviour of the $\Gamma$-function is that the kernel $k_{1}$ belongs to $\mathscr{L}_{\alpha}$ for $\alpha>-1$. 
For facts about the $\Gamma$-function needed in this article we refer to [1]. Also, the sequence $\lambda_{n}=\log p_{n}, n=1,2,3, \ldots$, is such that (8) holds. Then, in the case of the $f_{1}$-kernel, the essential part needed in order to have proved Theorem 1 is to establish the condition (2). Looking at the integral in (15), we have

$$
\int^{\infty} e^{-x / 2} \frac{q^{\prime \prime}(x)}{\sqrt{q^{\prime}(x)}} d x=\infty
$$

which, after integration by part, is equivalent to

$$
\int^{\infty} e^{-x / 2} \sqrt{q^{\prime}(x)} d x=\infty .
$$

We transform this condition to an equivalent one through $q=s(\exp (-\cdot))$ to get

$$
\int_{0} \sqrt{-s^{\prime}(t)} d t=\infty
$$

which is the condition in (2). The remaining condition (14) in Theorem 4 is equivalent to that in (3). As a result of Theorem 4 it is true that (12) holds, which is the same as (4). Thus we have obtained the proof of Theorem 1.

Let us continue with the next kernel $f_{2}$ or its transformed function $k_{2}$. Its Fourier transform $\hat{k}_{2}$ has a meromorphic extension to the complex plane and particularly it can be written as

$$
\hat{k}_{2}(w)=-\frac{i w \zeta(i w)}{2(2 \pi)^{i w-1} \sin \left(\frac{\pi}{2} i w\right)}, \quad w \in \mathrm{C},
$$

which satisfies

$$
\left(1 / \hat{k}_{2}\right)(-i)=0 \quad \text { and } \quad\left(1 / \hat{k}_{2}\right)(-2 m i)=0, \quad m=1,2,3, \ldots
$$

Besides these simple zeroes of $1 / \hat{k}_{2}$, it also has non-trivial singularities at all points where the function $w \mapsto \zeta(i w)$ has its non-trivial zeroes, i.e., in the strip $\mathfrak{s}(w) \in[-1,0]$. Particularly, $1 / \hat{k}_{2}$ is free from singularities in the strip $\mathfrak{R}(w) \in\left(-u_{o}, u_{o}\right)$, where $u_{o}=\min \left\{u>0 ; \zeta\left(\frac{1}{2}+i u\right)=0\right\}$. Thus the gap coefficient $c$ in (8) must satisfy $c>\pi / u_{o}$ from which it follows that $\delta>e^{\pi / u_{o}}$ in (5). For this coefficient $c$ condition (11) is satisfied. Furthermore, the function $1 / \hat{k}_{2}$ has an asymptotic behaviour as follows

$$
\left|\left(1 / \hat{k}_{2}\right)(u-i v)\right|=O(\exp (v \log 2 \pi)),
$$

for $|u| \leq C$ and $v \rightarrow \infty$, where $C$ is any finite constant. We can also see that $k_{2} \in \mathscr{L}_{\alpha}$ for $\alpha>-1$. As a reference for the properties of Riemann's $\zeta$-function we refer to [10]. Finally we have to check that the integral in (15) is 
equivalent to (6) in order to prove Theorem 2 . Thus, the remaining condition in this case is

$$
\int^{\infty} e^{-x} q^{\prime \prime}(x) d x=\infty .
$$

This condition can equivalently, by integration by part twice, be formulated as

$$
\int^{\infty} e^{-x} q(x) d x=\infty
$$

Expressed in term of the function $s=q(-\log (\cdot))$ the above integral transforms to the condition (6), i.e.

$$
\int_{0} s(t) d t=\infty
$$

This ends the proof of Theorem 2.

We conclude this section by considering the third kernel $f_{3}$. For this kernel we state the same theorem as in the case of the previous kernel. The Fourier transform $\hat{k}_{3}$ can be written as

$$
\hat{k}_{3}(w)=-\frac{i w\left(1-2^{1+i w}\right) \zeta(1+i w)}{2(2 \pi)^{i w} \sin \frac{\pi}{2}(1+i w)}, \quad w \in \mathrm{C} .
$$

The zeroes of $1 / \hat{k}_{3}$ are in the lower plane and given by

$$
\left(1 / \hat{k}_{3}\right)(-i-2 i m)=0, \quad m=0,1,2, \ldots
$$

Its interesting singularities, on the other hand, are given by the non-trivial zeroes of the function $w \mapsto \zeta(1+i w)$, i.e., those that are located in the strip $\Im(w) \in[0,1]$. As above, we have $1 / \hat{k}_{3}$ is free from singularities in the strip $\Re(w) \in\left(-u_{o}, u_{o}\right)$, where $u_{o}=\min \left\{u>0 ; \zeta\left(\frac{1}{2}+i u\right)=0\right\}$. The reciprocal Fourier transform has the asymptotic behaviour

$$
\left|\left(1 / \hat{k}_{3}\right)(u-i v)\right|=O(\exp (v \log \pi))
$$

for $|u| \leq C$ and $v \rightarrow \infty$, where $C$ is any finite constant. Also $k_{3} \in \mathscr{L}_{\alpha}$ for $\alpha>2$. We get the same integral divergence condition as in (39) above. Hence it follows that Theorem 2 also holds for this kernel. Thus, Theorem 1 and Theorem 2 together with its variant are proved and that completes this section. 


\section{REFERENCES}

1. Ahlfors, Lars V., Complex Analysis. Second Edition, McGraw Hill, New York 1966.

2. Fuchs, W. H. J., On the closure of $\left\{e^{-t} t^{a_{v}}\right\}$, Proc. Cambridge Philos. Soc. 42 (1946), 91-105.

3. Halász, Gábor, Remarks to a paper of D. Gaier on gap theorems, Acta Sci. Math. (Szeged) 28 (1967), 311-322.

4. Johansson, Bo, A general high indices theorem with an application to a conjecture by Rényi, Ark. Mat. 17 (1979), 279-286.

5. Johansson, Bo, Some distributional Tauberian theorems, Analysis 1 (1981), 197-208.

6. Johansson, Bo I., A Tauberian vanishing theorem, Anal. Math. 22 (1996), 25-34.

7. Korevaar, J., A very general form of Littlewood's theorem, Indag. Math. 16 (1954), 36-45.

8. Levinson, Norbert, Gap and Density Theorems, Amer. Math. Soc. Coll. Publ. 26, Amer. Math. Soc., New York 1940.

9. Ostrowski, Alexander, Über quasianalytische Funktionen und Bestimmtheit asymptotischer Entwickelungen, Acta Math. 53 (1929), 181-266.

10. Titchmarsh, E. C., The Theory of the Riemann Zeta-Function, Clarendon Press, Oxford 1951.

DEPARTMENT OF MATHEMATICAL SCIENCES

CHALMERS UNIVERSITY OF TECHNOLOGY

DEPARTMENT OF MATHEMATICAL SCIENCES

UNIVERSITY OF GOTHENBURG

SE-41296 GOTHENBURG

SWEDEN

E-mail: bo@chalmers.se 